\title{
Equilibrium gated radionuclide angiocardiography: Its invention, rise, and decline and ... comeback?
}

\author{
Frans J. Th. Wackers, MD, PhD, MASNC ${ }^{\mathrm{a}}$ \\ ${ }^{a}$ Yale University School of Medicine, New Haven, CT
}

Received Jan 13, 2016; accepted Jan 13, 2016

doi: 10.1007/s12350-016-0414-6

\section{See related article, pp. 348-361}

The seminal publication by Zaret and Strauss ${ }^{1}$ in 1971 on the assessment of cardiac left ventricular function without cardiac catheterization, using radionuclide cardiac blood pool imaging, marks the first appearance of nuclear cardiology in the clinical literature.

I believe that it is of interest to consider the history of the invention, the rapid initial clinical acceptance of equilibrium radionuclide angiocardiography (ERNA) for measuring left ventricular ejection fraction (LVEF), its subsequent decreasing clinical use, and now perhaps, its comeback.

Until 1971, blood pool imaging with Tc-99m-labeled human serum albumin (HSA) was used mostly to detect pericardial effusion. Zaret and Strauss' invention consisted of the acquisition of a series of short gamma camera images of the heart after Tc-99m-HSA had equilibrated in the intravascular space. Cardiac imaging was performed by briefly opening and closing an imaging gate on the gamma camera. The opening of the gate was triggered at predetermined delays after the electrocardiographic R-wave during systole and diastole, respectively. First, the imaging gate was set on the downslope of the T-wave for $40 \mathrm{~ms}$ to capture ventricular end-systole (ES). The opening and closing of the gate was repeated during 200-400 successive heartbeats.

Reprint requests: Frans J. Th. Wackers, MD, PhD, MASNC, Yale University School of Medicine, New Haven, CT, USA; frans.wackers@yale.edu

J Nucl Cardiol 2016;23:362-5.

1071-3581/\$34.00

Copyright (C) 2016 American Society of Nuclear Cardiology.
The scintillations were displayed on an oscilloscope and continuously exposed through open lens to photographic film or paper. Then, the gate was moved to perform a similar image acquisition during the last $60 \mathrm{~ms}$ of the cardiac cycle immediately before the next R-wave, thus capturing ventricular end-diastole (ED). The resulting summed ED and ES analog images were projected life size and contours of the LV were traced by hand. Left ventricular volumes and LVEF were determined using the planimetric area-length method routinely used in the cardiac catheterization laboratory.

Shortly after this publication, Secker-Walker et al, ${ }^{2,3}$ knowing that counts from radiolabeled blood were proportional to blood volume, acquired digital images of the heart in $32 \times 32$ matrix on an a DEC minicomputer PDP-12 with $8 \mathrm{~K}$ 12-bit word memory. Rather than acquiring ES and ED images sequentially, these investigators acquired simultaneously systolic and diastolic images from the same cardiac cycle using dual gating. Counts collected over 300 cardiac cycles were summed and integrated into final ED and ES digital images. This study also determined that the left anterior oblique view was optimal because at this angle RV and LV activity were separated naturally by the interventricular septum. These investigators also recognized the need for correction of non-cardiac background activity when calculating LVEF. Rather than making geometric assumptions, LVEF was now calculated from actual counts in ED and ES regions of interest (ROI): $\mathrm{LVEF}=$ counts in ED minus counts in ES, divided by background-corrected counts in ED.

Alpert et $\mathrm{al},{ }^{4}$ in order to obtain more accurate dynamic information, expanded image acquisition to 8 frames, encompassing the entire cardiac cycle. Display of LV counts vs. time showed a curve representing volume changes during the cardiac cycle. The advantage of this new approach was that ES was no longer guesstimated from the electrocardiogram, but defined by the frame with the lowest count density. 
A major leap forward was the work by Green et $\mathrm{al}^{5}$ who explored LIST-mode rather than frame-mode computer acquisition. Cardiac counts and R-wave signal were recorded in LIST-mode buffers on a $24 \mathrm{~K}$ core minicomputer. The data were then organized in two ways: low temporal resolution (12 frames per cardiac cycle) $32 \times 32$ digital images for movie display and a high temporal resolution (80-100 points per cardiac cycle) time-activity curve for precise determination of ES. Complete data collection, analysis, and display were accomplished in 2-3 minutes. This short acquisition time made it feasible not only to acquire ERNA at rest, but also during peak exercise; a feat never accomplished before.

In 1977, Strauss ${ }^{6}$ introduced a further improvement and simplification of image acquisition and analysis, called "MUGA" (multigated acquisition). All presentday computer hardware and software for gated cardiac blood pool imaging hark back to concepts incorporated in the original MUGA program. Before image acquisition is started, the RR interval is monitored and the average cardiac cycle is divided into 16-28 equal time intervals for multiple gating. The ECG R-wave is the synchronizing trigger for starting and restarting multigated image acquisition sequences. Imaging data acquired during each time interval are stored in frame mode in specific corresponding locations (bins) in the computer. Acquisition was usually terminated at preset time (2-3 minutes). The summed image data of all heartbeats in each bin are displayed as an endless loop movie: the "representative cardiac cycle." From an ROI over the LV, a time-activity curve can be generated to calculate count-based LVEF.

Subsequent refinements of processing software explored semi-automated ways to identify left ventricular edges, i.e., fixed vs. variable, ${ }^{7}$ manual vs. mathematical. The purpose of greater number of frames during the cardiac cycle was to improve temporal resolution required for precise identification of the point of ES and for measuring other dynamic parameters of LV function, such as peak ejection rate (PER) and peak filling rate (PFR). Multiple frames require multiple LV edge detections, which in turn may introduce new inaccuracies, reflected in ragged time-activity curves. Smoothing of the time-activity curve, although esthetically pleasing, is undesirable as it introduces further inaccuracies.

Without correction for non-cardiac background activity, LVEF is systematically underestimated. The initial method of correcting for non-cardiac blood activity was based on average count density in a crescent-shaped area postero-lateral of the LV. LV counts were then corrected pixel by pixel for average background activity.
In order to measure dynamic LV functions with greater accuracy, Lee et $\mathrm{al}^{8}$ in our laboratory introduced the concept of cycle-dependent background correction. Even after the above-mentioned standard background correction is applied, there are still residual non-ventricular counts in an area encompassed by the ED border and the ES border, the wall motion region. Counts in this expanding wall motion region show a smooth curve (cycle-dependent background curve) that increases during systole and decreases during diastole. The latter curve is then subtracted from the raw time-activity curve over the ED ROI resulting in a background-corrected LV time-activity curve. PER and PFR are then determined from the slopes of the final curve.

At the 1976 American Heart Association meeting in Miami, Borer et $\mathrm{al}^{9}$ presented for the first time the results of exercise gated blood pool imaging in patients with angiographic coronary artery disease. The presentation of ERNA movies, acquired during peak exercise, was received with considerable excitement by the audience. Exercise-induced worsening of global and regional LV function, suggesting ischemia, was demonstrated in real time. Around the same time, Thallium-201 became available for diagnostic stress myocardial perfusion imaging. But static myocardial perfusion images seemed now less exciting than movies of the beating heart. Initial publications on rest-exercise ERNA suggested very high sensitivity (95\%) and specificity (100\%) for detecting significant angiographic coronary artery disease,${ }^{10}$ higher than that reported for exercise Thallium-201 imaging. ${ }^{11}$ However, with increasing clinical experience, when more and less-selected patients were included in exercise ERNA studies, it became apparent that both sensitivity and specificity varied substantially depending on the patient population. ${ }^{12}$ Abnormal exercise LVEF response was not only observed in patients with coronary artery disease, but also in patients with other heart diseases, e.g., cardiomyopathy, valvular heart disease, or conduction abnormalities. Although exercise LV function testing had less diagnostic value for the detection of coronary artery disease than initially anticipated, peak exercise LVEF had important prognostic value in patients with variety of cardiac diseases.

Over the subsequent years, exercise ERNA was used less and less for diagnosing coronary artery disease. However, resting ERNA became well established as the method of choice for readily assessing LVEF, regional wall motion, and cardiac morphology. The imaging procedure was fully standardized and required only a few minutes of camera time, and LVEF values were highly reproducible.

When in the early 1980s echocardiography became a routine part of clinical cardiology, the use of resting 
ERNA decreased further. Echocardiographic equipment was smaller and more mobile; studies were performed under direct supervision of cardiologists. Even though it was shown repeatedly that ERNA LVEF was more reproducible than echocardiographic LVEF, ${ }^{13}$ the ease of access and greater flexibility of echocardiography laboratories favored widespread clinical use.

I believe that there was another reason why the clinical use of ERNA decreased. In the late 1970s and early 1980s, nuclear medicine departments were not sufficiently responsive to cardiologists' requests for prompt assessment of cardiac function. As a consequence, potential customers were lost and with it the clinical demand for ERNA withered. Additionally, with the advent of Tc-99m-labeled myocardial perfusion imaging agents, the focus of the nuclear cardiology community was directed at SPECT imaging. Planar imaging was no longer state of the art and became all but obsolete.

In recent years, novel dedicated cardiac SPECT cameras with multiple solid-state detectors, designed to acquire 3-dimensional image data, were introduced in clinical practice. These devices offer the benefit of improved count sensitivity, spatial and energy resolution, shortened imaging time, and reduced patient radiation exposure. The downside of this development was that laboratories no longer could provide time-tested planar imaging procedures, such as ERNA.

One year ago I wrote in this Journal: "I believe that ERNA should be considered the methodology of choice for sequential LVEF. Regrettably many laboratories have abandoned regular use of ERNAs or have limited experience." 14 A recent interrogation of the nuclear cardiology database of the Intersocietal Accreditation Commission confirms that of 3,645 nuclear laboratories that applied for accreditation, all sought accreditation for SPECT myocardial perfusion imaging, but only $21 \%$ applied for ERNA accreditation (IAC Baltimore personal communication).

Currently, the most common clinical indication for resting ERNA is serial assessment of LVEF in patients undergoing chemotherapy. Additional approved, but less frequently requested, clinical indications for ERNA are assessment of global and regional LV function in patients with recent or old infarction, in patients with congestive heart failure, and in patients who are potential candidates for implantation of defibrillator.

Although traditional NaI SPECT cameras have the capability of acquiring ECG-gated blood pool studies, the typical temporal resolution of 8-16 frames per cardiac cycle is suboptimal for measuring LVEF, PER, and PFR accurately.

In 2013, Welsh et $\mathrm{al}^{15}$ demonstrated that 3D 24frame gated SPECT data, acquired on novel dedicated cardiac cameras with Cadmium-Zinc-Telluride (CZT) detectors, can be re-projected forward onto a plane along the left anterior oblique (LAO) axis, thus creating a 24-frame gated planar LAO ERNA. The resulting gated planar images can be processed using standard planar ERNA analysis software.

In this issue of the Journal, Chen et $\mathrm{al}^{16}$ compared biventricular LVEF, ED, and ES volumes derived from conventional 24-frame gated planar ERNA with the same LV function parameters derived from 24-frame gated CZT SPECT ERNA and 24-frame gated re-projected CZT planar ERNA. They found good overall correlations (i.e., $r$ values) between planar ERNA for both CZT SPECT ERNA and CZT re-projected planar ERNA. However, by Bland-Altman analysis the limits of agreement between planar ERNA LVEF and CZT reprojected LVEF were substantially narrower than with CZT SPECT LVEF over the full range of LVEFs, suggesting that re-projection of 3D CZT gated data indeed may substitute planar ERNA.

Unfortunately, the authors limited the comparative analysis to calculating LVEF and LV volumes. They did not calculate PER and PFR from the 24 available frames. Had they used cycle-dependent background correction, these dynamic parameters could have been calculated as well.

Because of the complex morphology of the right ventricle (RV) and overlap with other cardiac chambers, reliable assessment of RVEF since long has been recognized as being problematic using planar ERNA. For this reason, the 2009 ASNC guidelines discourage the use of planar ERNA for assessment of RVEF and recommend using the radionuclide first pass or gated first pass technique as a potential better approach to assess RVEF. ${ }^{17}$ Although I am quite comfortable using planar ERNA LVEF as a "second best gold standard" after MRI, I believe that Chen's study does not provide convincing data concerning the validity of assessment of RV function without MRI as a comparator. The BlandAltman analysis of RV parameters shows suboptimal agreements between CZT SPECT and planar ERNA. It is conceivable that a comparison of MRI-derived RVEF with CZT re-projected RVEF would have shown different results.

Nevertheless, the results of Chen's study are of substantial clinical interest.

I agree with the conclusion that re-projection of 3D data onto a 2D plane may allow for substituting planar ERNA imaging. This approach has the advantage that a well-validated imaging procedure and analysis of LV function may find continued clinical use. Using CZT cardiac SPECT cameras, image acquisition can be shortened and patient radiation exposure reduced. Because of this, I am hopeful that ERNA may make a comeback in the nuclear cardiology laboratories. 


\section{References}

1. Strauss HW, Zaret BL, Hurley PJ, Natarajan TK, Pitt B. A scintigraphic method for measuring left ventricular ejection fraction in man without cardiac catheterization. Am J Cardiol 1971;28:575-80

2. Parker JA, Secker-Walker R, Hill R, Siegel BA, Potchen EJ. A new technique for the calculation of left ventricular ejection fraction. J Nucl Med 1972;13:649-51.

3. Secker-Walker RH, Resnick L, Kunz H, Parker JA, Hill RL, Potchen EJ. Measurement of left ventricular ejection fraction. J Nucl Med 1973;14:798-802.

4. Alpert NM, McKusick KA, Pohost GM, Dinsmore RE, Potsaid MS. Noninvasive nuclear kinecardiography. J Nucl Med 1974;15:1182-4.

5. Green MV, Ostrow HG, Douglas MA, Meyers RW, Scott RN, Bailey JJ, Johnston GS. High temporal resolution ECG-gated scintigraphic angiocardiography. J Nucl Med 1975;16:95-8.

6. Burow RD, Strauss HW, Singleton R, Pond M, Rehn T, Bailey IK, Griffith LC, Nickoloff E, Pitt B. Analysis of left ventricular function from multiple gated acquisition cardiac blood pool imaging. Comparison to contrast angiography. Circulation 1977;56:1024-8

7. Sorenson SG, Hamilton GW, Williams DL, Ritchie JL. R-wave synchronized blood pool imaging: A comparison of the accuracy and reproducibility of fixed and computer-automated varying regions-of-interest for determining the left ventricular ejection fraction. Radiology 1979;131:473-8.

8. Lee FA, Fetterman R, Zaret BL, Wackers FJTh. Rapid radionuclide-derived systolic and diastolic cardiac function using cycledependent background correction and Fourier analysis. In: Proceedings of computers in cardiology. IEEE Computer Society. 811Sept 1985. p. 443-6.

9. Borer JS, Bacharach SL, Green MV, Kent KM, Epstein SE, Johnston GS. Real-time radionuclide cineangiography in the noninvasive evaluation of global and regional left ventricular function at rest and during exercise in patients with coronary artery disease. New Engl J Med 1977;296:839-44.

10. Borer JS, Kent KM, Bacharach SL, Green MV, Rosing DR, Seides SF, Epstein SE, Johnston GS. Sensitivity, specificity and predictive accuracy of radionuclide cineangiography during exercise in patients with coronary artery disease. Comparison with exercise electrocardiography. Circulation 1979;60:572-80.

11. Ritchie JL, Zaret BL, Strauss HW, Pitt B, Berman DS, Schelbert HR, Ashburn WL, Berger HJ, Hamilton GW. Myocardial imaging with Thallium-201: a multicenter study in patients with angina pectoris or acute myocardial infarction. Am $\mathrm{J}$ Cardiol 1978;42:345-50.

12. Rozanski A, Diamond GA, Berman DS, Forrester JS, Morris D, Swan HJ. The declining specificity of exercise radionuclide ventriculography. New Engl J Med 1983;309:518-22.

13. Van Royen N, Jaffe CC, Krumholz HK, Johnson KM, Lynch PJ, Natale D, Atkinson P, Deman P, Wackers FJTh. Comparison and reproducibility of visual echocardiographic and quantitative radionuclide left ventricular ejection fraction. Am J Cardiol 1996;77:843-50.

14. Wackers FJTh. Has LVEF changed beyond chance? Limits of agreement of radiotracer-derived LVEF. J Nucl Cardiol 2015;22:1244-6.

15. Wells RG, Marvin B, Kovalski G, Ruddy TD. Planar radionuclide angiography with a dedicated cardiac SPECT camera. J Nucl Cardiol 2013;20:358-66.

16. Chen YC, Ko CL, Yen RF, Lo MF, Huang YH, Hsu PY, Wu YW, Cheng MF. Comparison of biventricular ejection fractions using cadmium-zinc-telluride SPECT and planar equilibrium radionuclide angiography. J Nucl Cardiol 2016. doi:10.1007/s12350015-0367-1.

17. Corbett JR, Akinboboye OO, Bacharach SL, Borer JS, Botvinick EH, Depuey EG, Ficaro EP, Hanen CL, Henzlova MJ. ASNC imaging guidelines for nuclear cardiology procedures. Equilibrium radionuclide angiocardiography. http://www.asnc.org/guidelines andstandards. Accessed 11 Jan 2016. 